\title{
Limiting Statistics of the Largest and Smallest Eigenvalues in the Correlated Wishart Model
}

\author{
Tim Wirtz ${ }^{1 \dagger}$, Mario Kieburg ${ }^{\ddagger 2}$ and Thomas Guhr ${ }^{1 *}$ \\ ${ }^{1}$ Fakultät für Physik, Universität Duisburg-Essen, 47048 Duisburg, Germany \\ ${ }^{2}$ Fakultät für Physik, Universität Bielefeld, 33501 Bielefeld, Germany
}

(Dated: September 30, 2018)

\begin{abstract}
The correlated Wishart model provides a standard tool for the analysis of correlations in a rich variety of systems. Although much is known for complex correlation matrices, the empirically much more important real case still poses substantial challenges. We put forward a new approach, which maps arbitrary statistical quantities, depending on invariants only, to invariant Hermitian matrix models. For completeness we also include the quaternion case and deal with all three cases in a unified way. As an important application, we study the statistics of the largest eigenvalue and its limiting distributions in the correlated Wishart model, because they help to estimate the behavior of large complex systems. We show that even for fully correlated Wishart ensembles, the TracyWidom distribution can be the limiting distribution of the largest as well as the smallest eigenvalue, provided that a certain scaling of the empirical eigenvalues holds.
\end{abstract}

PACS numbers: 05.45.Tp, 02.50.-r, 02.20.-a

Time series analysis yields rich information about the dynamics but also about the correlations in numerous systems in physics, climate research, biology, medicine, wireless communication, finance and many other fields [1-12]. Suppose we have a set of $p$ time series $M_{j}, j=1, \ldots, p$ of $n(n \geq p)$ time steps each, which are normalized to zero mean and unit variance. The entries are real, complex, quaternion, i.e. $M_{j}(t) \in \mathbb{R}, \mathbb{C}, \mathbb{H}$, for $\beta=1,2,4$ and $t=1, \ldots, n$. We arrange the time series as rows into a rectangular data matrix $M$ of size $p \times n$. The empirical correlation matrix of these data,

$$
C=\frac{1}{n} M M^{\dagger},
$$

with $\uparrow$ the Hermitian conjugation, is positive definite and either real symmetric, Hermitian, or Hermitian self-dual for $\beta=1,2,4$ and measures the linear correlations between the time series.

The largest and the smallest eigenvalue of a correlation matrix are highly relevant in many fields. In a simple, interacting dynamical system [13, 14], occurring in physics [15], biology [16], chemistry [17, ecology [18, etc., the cumulative distribution function of the largest eigenvalue estimates the probability to find the system in a stable regime [15. In high dimensional statistical inference, linear principal component analysis is a method to reduce the dimension of the observations to "significant directions" 19. Especially, the largest eigenvalue corresponds to the most "significant" component [19-22]. Another example is factor analysis, where the largest eigenvalue can be used to study common properties 19. The ratio of largest and smallest eigenvalue is important for the statistics of the condition number [23, 24, in numerical analysis including large random matrices. In wireless communication, eigenvalue based detection [25 27] is a promising technique for spectrum sensing in cognitive radio. It utilize the statistics of the ratio of largest and smallest eigenvalue to estimate certain statistical tests 28 30. The smallest eigenvalue is important for estimates of the error of a received signal [31 33 in wireless communication, for estimates in linear discriminant 34] as well as in principal component analysis [4, it is most sensitive to noise in the data 4 and crucial for the identification of single statistical outliers [5. In finance, it is related to the optimal portfolio [35].

These examples show the considerable theoretical and practical relevance to study the distributions $\mathcal{P}_{\max }^{(\beta)}(t)$, $\mathcal{P}_{\min }^{(\beta)}(s)$ of the largest, respectively, smallest eigenvalue. Both quantities can be traced back to gap probabilities, namely

$$
\begin{aligned}
& \mathcal{P}_{\max }^{(\beta)}(t)=\frac{\mathrm{d}}{\mathrm{d} t} E_{p}^{(\beta)}([0, t] ; p), \\
& \mathcal{P}_{\min }^{(\beta)}(s)=-\frac{\mathrm{d}}{\mathrm{d} s} E_{p}^{(\beta)}([0, s] ; 0),
\end{aligned}
$$

where $E_{p}^{(\beta)}([a, b] ; m)$ is the probability to find $m$ out of $p$ eigenvalues in the interval $[a, b]$.

This article has three major goals: First, we provide for the first time a framework to map a large class of invariant observables in correlated Wishart ensembles to invariant matrix models. Second, we explicitly apply this framework to the cumulative distribution function (2) of the largest eigenvalue and find an invariant matrix model. Third, we show that for a certain class of $C$ 's, $p / n$ fixed and $n, p$ tending to infinity the largest, respectively, smallest eigenvalue are Tracy-Widom distributed.

The ensemble of random Wishart correlation matrices $W W^{\dagger} / n$ [19, 20] consists of $p \times n$ model data matrices $W \in \operatorname{Mat}_{p \times n}(\mathbb{K})$, where $\mathbb{K}=\mathbb{R}, \mathbb{C}$ or $\mathbb{H}$ for $\beta=1,2,4$, such that upon average $\left\langle W W^{\dagger} / n\right\rangle=C$. Data analysis strongly corroborate, see e.g. Refs. [3, 7-11, 36, the 
Gaussian Wishart model [19, 20],

$$
P(W \mid C) \sim \exp \left(-\frac{\beta}{2} \operatorname{tr} W W^{\dagger} C^{-1}\right) .
$$

The matrix $W W^{\dagger} / n$ is known as Wishart correlation matrix. The corresponding measure $\mathrm{d}[W]$ and all other measures $\mathrm{d}[\cdot]$ occurring later on are flat, i.e., the products of the independent differentials. Due to the invariance of $\mathrm{d}[W]$, invariant observables depend on average solely on the distinct, always non-negative eigenvalues $\Lambda_{j}, j=1, \ldots, p$ of $C$ which are referred to as the empirical ones. We arrange them in the diagonal matrix $\hat{\Lambda}=\Lambda \otimes \mathbb{1}_{\gamma_{2}}$ and introduce $\gamma_{2}=1$ if $\beta=1,2$ and $\gamma_{2}=2$ if $\beta=4$ and for later purpose $\gamma_{1}=2 \gamma_{2} / \beta$, where $\mathbb{1}_{N}$ is the unity matrix in $N$ dimensions.

We consider an observable $\mathcal{O}\left(W W^{\dagger}\right)$ which is invariant under an arbitrary change of basis and $\mathcal{O}\left(W W^{\dagger}\right)=$ $\mathcal{O}\left(W^{\dagger} W\right)$. This is a very weak assumption when studying the eigenvalue statistics of $W W^{\dagger}$. We are interested in the average

$$
\left\langle\mathcal{O}\left(W W^{\dagger}\right)\right\rangle=K \int \mathrm{d}[W] \mathcal{O}\left(W W^{\dagger}\right) P(W \mid \hat{\Lambda}),
$$

where the integration domain is $\operatorname{Mat}_{p \times n}(\mathbb{K})$ and $K$ is a normalization constant. The non-triviality of the integral (5) is due to a group integral of the form

$$
\begin{aligned}
& \Phi_{\beta}\left(X, \hat{\Lambda}^{-1}\right)= \\
& \int \mathrm{d} \mu(V) \exp \left(-\frac{\beta}{2} \operatorname{tr} V\left(X \otimes \mathbb{1}_{\gamma_{2}}\right) V^{\dagger} \hat{\Lambda}^{-1}\right),
\end{aligned}
$$

where $\mathbb{1}_{N}$ is $N$ dimensional unit matrix, the integration domain is $\mathrm{O}(p), \mathrm{U}(p)$ or $\mathrm{USp}(2 p)$ for $\beta=1,2,4$, respectively, and $X=\operatorname{diag}\left(x_{1}, \ldots, x_{p}\right)$ are the distinct eigenvalues of $W W^{\dagger}=V\left(X \otimes \mathbb{1}_{\gamma_{2}}\right) V^{\dagger}$. It is known as the orthogonal, unitary or unitary-symplectic Itzykson-Zuber integral [37. For the unitary case only, it can be computed analytically and is given in a closed form [37 39.

We replace the invariants of $W W^{\dagger}$ in Eq. (5) by those of the $n \times n$ matrix $W^{\dagger} W$. Thus, after introducing a $\delta$-function and replacing $W^{\dagger} W$ by a matrix in the same symmetry class, say $Q$, we find

$$
\begin{array}{r}
\left\langle\mathcal{O}\left(W W^{\dagger}\right)\right\rangle=K \int \mathrm{d}[H, Q] \mathcal{O}(Q) \exp (\imath \operatorname{tr} H Q) \\
\times \int \mathrm{d}[W] \exp \left(-\imath \operatorname{tr} H W^{\dagger} W\right) P(W \mid \hat{\Lambda})
\end{array}
$$

where the integral of $Q$ and $H$ is over the set of real symmetric, Hermitian, Hermitian self-dual matrices of dimension $n \times n$ for $\beta=1,2,4$, respectively. The $H$ integral is the Fourier representation of the delta function. A detailed mathematical discussion will be given elsewhere 40. The advantage of this approach is that $H$ couples to $W^{\dagger} W$ while $\Lambda^{-1}$ to $W W^{\dagger}$, see Eq. (4). Hence the integral over $H$ is invariant under $H \rightarrow U H U^{-1}$ with $U$ orthogonal, unitary, and unitary symplectic for $\beta=1,2,4$ respectively. The remaining $W$ integral becomes a Gaussian integral over an $n p$-dimensional vector with entries in $\mathbb{K}$, yielding

$$
\left\langle\mathcal{O}\left(W W^{\dagger}\right)\right\rangle=\int \mathrm{d}[H] \frac{\mathcal{F}_{n}[\mathcal{O}](H)}{\operatorname{det}^{1 / \gamma_{1}}\left(\mathbb{1}_{n p \gamma_{2}}+\imath H \otimes \frac{2}{\beta} \Lambda\right)} .
$$

In the expression (8), we introduce the Fourier transform of the observable $\mathcal{O}$

$$
\mathcal{F}_{n}[\mathcal{O}](H)=\frac{1}{(2 \pi)^{\mu}} \int \mathrm{d}[Q] \mathcal{O}(Q) \exp (\imath \operatorname{tr} H Q)
$$

where $\mu=n(n+1) / 2, n^{2}, n(2 n-1)$ is the number of real degrees of freedom of $Q$ for $\beta=1,2,4$, respectively. If we know $\mathcal{F}_{n}[\mathcal{O}](H)$, we can express the average (5) as an invariant matrix integral. Thereby we completely outmaneuver the Itzykson-Zuber integral (6).

We exploit this general observation to the statistics of the extreme eigenvalues. The gap probabilities in Eqs. (2) and (3) can be written as an ensemble averaged observable. We carry it out for Eq. (2) only, since for Eq. (3) it works analogously. The joint eigenvalue distribution function derived from Eq. (4) is

$$
P(X \mid \Lambda)=K_{p \times n}\left|\Delta_{p}(X)\right|^{\beta} \operatorname{det}^{v} X \Phi_{\beta}\left(X, \hat{\Lambda}^{-1}\right),
$$

with normalization constant $K_{p \times n}$, Vandermonde determinant $\Delta_{p}(X)=\prod_{i<j}\left(x_{j}-x_{i}\right)$ and $v=\beta(n-p+1-$ $2 / \beta) / 2$, see Ref. 41, 42. As $\Phi_{2}$ is known, in the complex case the joint probability distribution function provides a representation that can be handled analytically [39]. The highly non-trivial part is the group integral Eq. (6.) The gap probability to find all eigenvalues below $t$ can then be cast into the form

$$
\begin{aligned}
E_{p}^{(\beta)}([0, t] ; p) & =K_{p \times n} \int \mathrm{d}[X]\left|\Delta_{p}(X)\right|^{\beta} \operatorname{det}^{v} X \\
& \times \prod_{i=1}^{p} \Theta\left(t \mathbb{1}_{p}-x_{i}\right) \Phi_{\beta}\left(X, \hat{\Lambda}^{-1}\right),
\end{aligned}
$$

where $\Theta\left(x_{i}\right)$ is the Heaviside $\Theta$-function of scalar argument. The Heaviside function of matrix argument is known in terms of an Ingham-Siegel integral, see Ref. 43 and references therein. It is unity if its argument is positive definite and vanishes otherwise. Positive definiteness is an invariant property implying that the $\Theta$-function depends on the eigenvalues $a_{i}$ of $A$ only,

$$
\Theta(A)=\Theta(a)=\prod_{i=1}^{N} \Theta\left(a_{i}\right)
$$

Since the integral (11) is over the whole spectrum of $W W^{\dagger}$, we express the gap probability as averaged $\Theta$ - 
function,

$$
\begin{aligned}
E_{p}^{(\beta)}([0, t] ; p) & =K_{p \times n} t^{n p \beta / 2} \int \mathrm{d}[W] P(W \mid \sqrt{t} \hat{\Lambda}) \\
& \times \Theta\left(\mathbb{1}_{\gamma_{2} p}-W W^{\dagger}\right)
\end{aligned}
$$

where $W \in \operatorname{Mat}_{p \times n}(\mathbb{K})$. Analogously, the gap probability (3) is given by

$$
\begin{array}{r}
E_{p}^{(\beta)}([0, s] ; 0)=K_{p \times n} s^{n p \beta / 2} \int \mathrm{d}[\widehat{W}] P(\widehat{W} \mid \sqrt{s} \hat{\Lambda}) \\
\times \Theta\left(\widehat{W} \widehat{W}^{\dagger}-\mathbb{1}_{\gamma_{2} p}\right) \operatorname{det}^{(n-p) / \gamma_{1}} \widehat{W} \widehat{W}^{\dagger},
\end{array}
$$

with $\widehat{W}$ is a square $p \times p$ matrix .

To employ our approach to the gap probability (13), we choose the observable to be $\mathcal{O}\left(W W^{\dagger}\right)=\Theta\left(\mathbb{1}_{\gamma_{2} p}-W W^{\dagger}\right)$. The matrix $\mathbb{1}_{\gamma_{2} n}-W^{\dagger} W$ has $p$ distinct eigenvalues that coincide with those of $\mathbb{1}_{\gamma_{2} p}-W W^{\dagger}$ and $n-p$ distinct eigenvalues that are exactly one. Hence, using Eq. (12) it is evident that $\mathcal{O}\left(W W^{\dagger}\right)=\mathcal{O}\left(W^{\dagger} W\right)$. To exchange the $Q, H$ and the $W$ integral, we shift the contour of $H$ by $-\imath \mathbb{1}_{\gamma_{2} n}$ and find the inverse Fourier-Laplace transform in Eq. (8)

$$
\mathcal{F}_{n}[\Theta]\left(H-\imath \mathbb{1}_{\gamma_{2} n}\right) \sim \frac{\exp \left(\operatorname{tr}\left(\imath H+\mathbb{1}_{\gamma_{2} n}\right)\right)}{\operatorname{det}^{\alpha / \gamma_{1}}\left(\imath H+\mathbb{1}_{\gamma_{2} n}\right)},
$$

where $\alpha=n-1+2 / \beta$. If we diagonalize $H=$ $U\left(Y \otimes \mathbb{1}_{\gamma_{2}}\right) U^{\dagger}$, where $U$ is in one of the three groups and $Y=\operatorname{diag}\left(y_{1}, \ldots, y_{n}\right)$ is the matrix of distinct eigenvalues of $H$, we arrive at a remarkable, new expression for the gap probability 11 .

$$
\begin{aligned}
& E_{p}^{(\beta)}([0, t] ; p)=K_{p \times n} \int_{\mathbb{R}^{n}} \frac{\mathrm{d}[Y]\left|\Delta_{n}(Y)\right|^{\beta}}{\operatorname{det}^{\alpha_{\beta} \beta / 2}\left(\imath Y+\mathbb{1}_{n}\right)} \\
& \times \frac{\exp \left(\gamma_{2} \operatorname{tr}\left(\imath Y+\mathbb{1}_{n}\right)\right)}{\prod_{k=1}^{p} \operatorname{det}^{\beta / 2}\left(\mathbb{1}_{n}+\left(\imath Y+\mathbb{1}_{n}\right) 2 \Lambda_{k} / t \beta\right)} .
\end{aligned}
$$

Likewise, we derive an invariant matrix model for the gap probability 13 .

$$
\begin{aligned}
& E_{p}^{(\beta)}([0, s] ; 0)=K_{p \times n} \int_{\mathbb{R}^{p}} \mathrm{~d}[Y]\left|\Delta_{p}(Y)\right|^{\beta} \\
& \times \frac{\mathcal{F}_{p}\left[\Theta\left(Q-\mathbb{1}_{\gamma_{2} p}\right) \operatorname{det}^{(n-p) / \gamma_{1}} Q\right](Y)}{\prod_{k=1}^{p} \operatorname{det}^{\beta / 2}\left(\mathbb{1}_{p}+\imath 2 \Lambda_{k} Y / s \beta\right)} .
\end{aligned}
$$

The Fourier integral can be done using the differential operator constructed in appendix B of Ref. [44, but the expression becomes cumbersome and we do not need these details for the following discussion.

Both results (16) and (17) have a $p$-fold product of determinants in the denominator in common. Due to the exponent $\beta / 2$, this eigenvalue integral can be studied, at least for $\beta=2,4$, using standard techniques of random

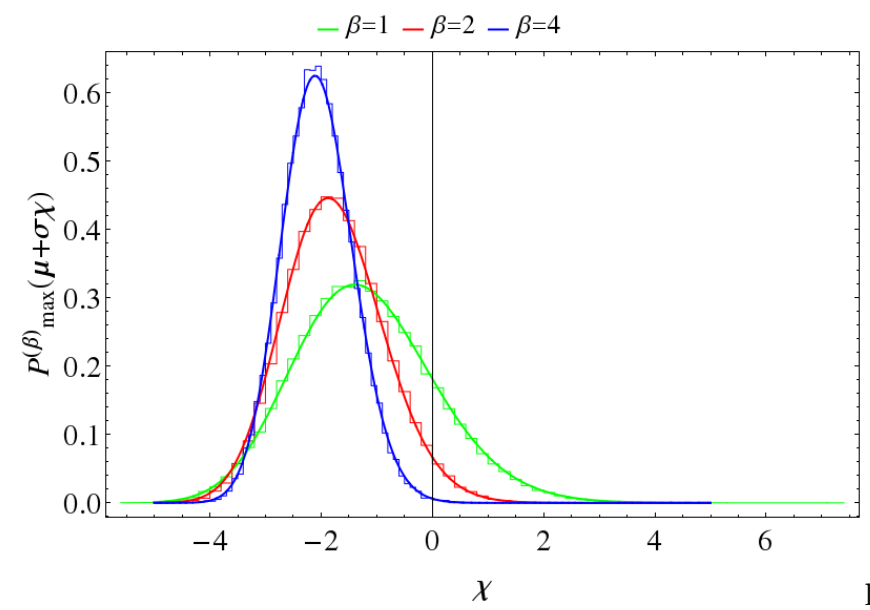

FIG. 1. Comparison of analytic results for the largest eigenvalue distribution (straight lines) with numerical simulations (symbols) for $\beta=1,2,4$. We consider 80000 realizations of $100 \times 300$ rectangular matrices, according to the distribution Eq. (4.).

matrix theory. For $\beta=1$ standard methods do not apply as square roots of characteristic polynomials appear. We further evaluate the exact expression (16) elsewhere [40]. Here we focus on the limiting behavior which is more relevant in applications. To this end, we assume that the empirical eigenvalues are random variables according to the distribution $\rho_{\mathrm{emp}}(\Lambda)$ and $t=\mu(\Lambda)+\sigma(\Lambda) \chi$, where the centering and scaling parameters $\mu(\Lambda)$ and $\sigma(\Lambda)$ are assumed to be large. We will show that this is a justified assumption.

For the uncorrelated Wishart ensemble, i.e. $\Lambda=\mathbb{1}_{p}$, previous works $[22,23,45,58$ focus on the exact as well as the limiting distribution of the largest eigenvalue $x_{\max }$ and the smallest eigenvalue $x_{\min }$ of $W W^{\dagger}$. For $n, p$ tending to infinity, while $p / n=\gamma^{2}$ is fixed, it was proved that the limiting distribution of $\chi_{\max }=\left(x_{\max }-\mu_{+}\right) \sigma_{+}^{-1}$ and $\chi_{\min }=\left(x_{\min }-\mu_{-}\right) \sigma_{-}^{-1}$ is the Tracy-Widom law $f_{\beta}(\chi)$ [59 61, where

$$
\sigma_{ \pm}= \pm \frac{(1 \pm \gamma)^{4 / 3}}{\gamma^{1 / 3}} n^{1 / 3} \text { and } \mu_{ \pm}=(1 \pm \gamma)^{2} n
$$

$\nu=n-p=\left(1-\gamma^{2}\right) n$ and $\gamma$ fixed for $p \rightarrow \infty$ [22]. Moreover, if $n, p$ tend to infinity, while $n-p$ is fixed it was shown that the limiting largest eigenvalue distribution is still Tracy-Widom [22, 47, 49].

For the correlated Wishart ensemble, the limiting largest eigenvalue distribution is known for $\beta=2$ in general [62, 63] and for $\beta=1,4$ solely when $\Lambda$ is a rank one perturbation of the identity matrix [55, 64, 65]. The smallest eigenvalue distribution was already studied in great detail in the microscopic limit, i.e. $n, p \rightarrow \infty$ while $n-p=\nu$ fixed in Refs. 41, 42, whereas for $n, p \rightarrow \infty$ with $p / n$ fixed no results are available yet.

Similar to Refs. [41, 42, we assume that the empiri- 
cal eigenvalues $\Lambda_{k}$ are of order $\mathcal{O}(1)$ for $n, p$ tending to infinity. It turns out that only the rescaled trace, $\langle\cdot\rangle_{\mathrm{s}}=$ $p^{-1} \operatorname{tr}(\cdot)$, of $\Lambda^{m}$, where $m=1$, does not tend to zero. Moreover, another simple estimate shows $\left\langle\Lambda^{m}\right\rangle_{\mathrm{s}} \sim \mathcal{O}(1)$ such that we cannot determine the exact leading order of the empirical eigenvalue variance $\operatorname{Var}_{s}(\Lambda)$. Consequently, we impose another requirement on the empirical eigenvalue distribution, namely $\operatorname{Var}_{\mathrm{s}}(\Lambda) \sim \mathcal{O}\left(1 / p^{\alpha}\right)$, where $\alpha>0$ is a free parameter which we fix later. As a consequence of the Tschebyscheff inequality,

$$
\mathbb{P}\left(\left|\Lambda-\langle\Lambda\rangle_{\mathrm{s}}\right| \geq x\right) \leq \frac{\operatorname{Var}_{\mathrm{s}}(\Lambda)}{x^{2}} \sim \mathcal{O}\left(1 / p^{\alpha}\right),
$$

we make the following ansatz for the empirical eigenvalues by

$$
\Lambda_{k}=\bar{\Lambda}+p^{-\alpha} \Lambda_{k}^{(1)}
$$

where $\bar{\Lambda}=\langle\Lambda\rangle_{\mathrm{s}}$ and $\Lambda_{k}^{(1)} \sim \mathcal{O}(1)$. If $C$ is a properly normalized correlation matrix, then $\bar{\Lambda}=\langle\Lambda\rangle_{\mathrm{s}}=1$.

Substituting Eq. 20 into Eq. (16), and expanding the $p$-fold product to leading order, under the assumption that $t$ is large, we find for each integration variable $y_{i}$

$$
\begin{aligned}
& \prod_{k=1}^{p} \frac{1}{\left(1+\left(\imath y_{i}+1\right) \frac{2}{t \beta}\left(\bar{\Lambda}+p^{-\alpha} \Lambda_{k}^{(1)}\right)\right)^{\beta / 2}} \\
& =\left(1+\frac{t \operatorname{tr} \Lambda^{(1)}}{p^{\alpha} p \bar{\Lambda}} \frac{\mathrm{d}}{\mathrm{d} t}+\ldots\right) \frac{1}{\left(1+\left(\imath y_{i}+1\right) \frac{2 \bar{\Lambda}}{t \beta}\right)^{p \beta / 2}},
\end{aligned}
$$

where $i=1, \ldots, n$. The dots correspond to higher powers of $p^{-\alpha}$ times the derivative $t \mathrm{~d} / \mathrm{d} t$. If we insert this expansion back into the cumulative distribution function (16) and keep only the leading terms in $n, p$ we find

$$
\begin{aligned}
E_{p}^{(\beta)}([0, t] ; p) & =\left.E_{p}^{(\beta)}([0, t] ; p)\right|_{\Lambda=\bar{\Lambda} \mathbb{1}_{p}} \\
& -\left.\frac{\operatorname{tr} \Lambda^{(1)}}{p^{\alpha} p \bar{\Lambda}} t \frac{\mathrm{d}}{\mathrm{d} t} E_{p}^{(\beta)}([0, t] ; p)\right|_{\Lambda=\bar{\Lambda} \mathbb{1}_{p}}+\ldots
\end{aligned}
$$

The first term on the right hand side of Eq. 22 is Eq. (11) for an uncorrelated Wishart ensemble with variance $\Lambda$. From the discussion above Eq. (18), we conclude that if we center and rescale appropriately, the first term in Eq. 22 converges to the integrated distribution function $F_{\beta}(\chi)=\int_{-\infty}^{\chi} f_{\beta}\left(\chi^{\prime}\right) \mathrm{d} \chi^{\prime}$, found by Tracy and Widom. Therefore, we focus our discussion on the second term in Eq. 22.

For the centered and rescaled threshold parameter $t=\mu_{+} \bar{\Lambda}+\sigma_{+} \bar{\Lambda} \chi$, we take $t$ times the derivative with respect to $t$ of a function, which in the limit $n, p \rightarrow \infty$ and either $n / p$ or $n-p$ fixed converges to $F_{\beta}(\chi)$. Due to $p^{-1} \operatorname{tr} \Lambda^{(1)} \rightarrow$ const. for $p \rightarrow \infty$, the prefactor is of or$\operatorname{der} \mathcal{O}\left(p^{-\alpha}\right)$. Solely the rescaling of the derivative $t \mathrm{~d} / \mathrm{d} t$

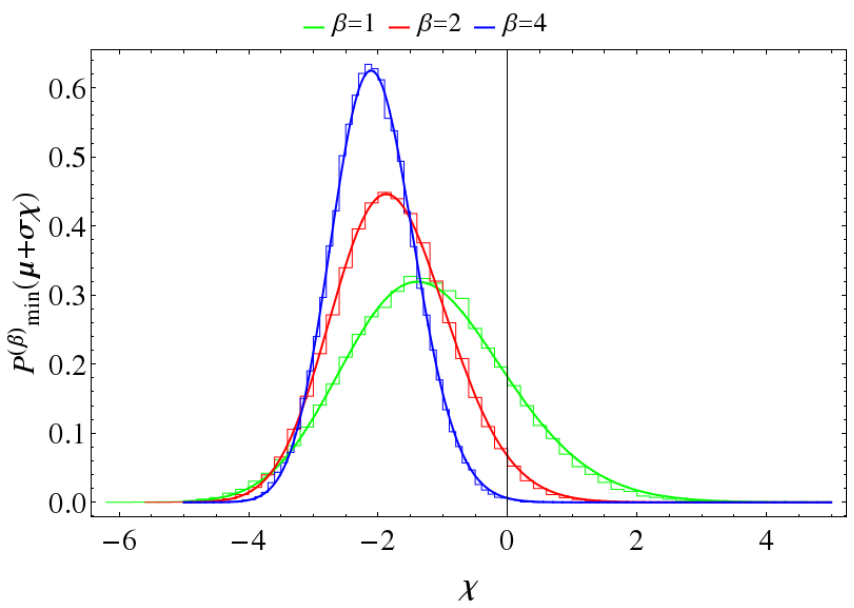

FIG. 2. Comparison of analytic results for the smallest eigenvalue distribution (straight lines) with the same numerical simulations (symbols) as in Fig. 1 for $\beta=1,2,4$.

can influence this order. A careful analysis shows if $\alpha$ is chosen such that for $n \rightarrow \infty$

$$
\frac{1}{p^{\alpha}} \frac{\mu_{+}}{\sigma_{+}}=\gamma^{2 \alpha-1}(1+\gamma)^{2 / 3} n^{2 / 3-\alpha} \rightarrow 0,
$$

the second term in Eq. 22 goes to zero as well. Thus, we require that $\alpha>2 / 3$ so that a macroscopic distance between the largest eigenvalue and the empirical eigenvalues is guaranteed only if the fluctuations of the empirical eigenvalues do not overlap with those of the largest eigenvalue.

Like the cumulative distribution function of the largest eigenvalue (16), the dependence of the smallest eigenvalue gap probability (17) on the empirical eigenvalues $\Lambda$ solely enter in the determinant in the denominator. Hence, we can apply the analysis done for the gap probability corresponding to the largest eigenvalue to that of the smallest one. Eventually, after centering and rescaling the threshold parameter, $s=\tilde{\mu}_{-} \bar{\Lambda}+\tilde{\sigma}_{-} \bar{\Lambda} \chi$, where $\tilde{\mu}_{-}$ and $\tilde{\sigma}_{-}$are as in Eq. (18) and assuming the same restrictions on the empirical eigenvalue distribution as above, we obtain that cumulative distribution function is $F_{\beta}(\chi)$.

To illustrate our findings, we compare our analytical results with Monte Carlo simulations for $\gamma \approx 0.33$. The empirical eigenvalues are random variables with respect to a uniform distribution such that $\operatorname{Var}_{s}(\Lambda)=p^{-7 / 4}$, $\langle\Lambda\rangle_{s}=1=\bar{\Lambda}$ and $n^{2 / 3} \operatorname{Var}_{s}(\Lambda) \approx 0.013 \ll 1$. The Comparison for the largest and the smallest eigenvalue distribution is shown in Fig. 1 and Fig. 2 respectively. To demonstrate the agreement with the numerical simulations, we properly adjust the centering without changing the limit behavior. For the smallest eigenvalue we even properly adjust the scaling by a constant shift of the order $\mathcal{O}(1 / n)$. This is because the smallest eigenvalue always "feels" the presents of a hard wall at zero, whereas the largest eigenvalue does not see any barrier such that the 
$1 / n$ correction is stronger for the smallest eigenvalue.

In conclusion, we presented a new approach to map observables depending on the eigenvalues of a Wishart matrix only, to an invariant Hermitian matrix model. We demonstrated the concept by applying it to the gap probabilities corresponding to the largest and smallest eigenvalue distributions. Utilizing these invariant matrix model, we showed that for special empirical eigenvalue spectra, the Tracy-Widom distribution persist for the smallest and the largest eigenvalue if $n, p$ tend to infinity while $p / n=\gamma^{2}$ is fixed. We confirmed our findings by numerical simulations.

A simultaneous but independent study on related issues was very recently put forward in Ref. [66].

We thank the Deutsche Forschungsgemeinschaft, Sonderforschungsbereich TR12 (T.W and T.G.) and the Alexander von Humboldt-Foundation (M.K.) for support.

* thomas.guhr@uni-due.de

${ }^{\dagger}$ tim.wirtz@uni-due.de

${ }^{\ddagger}$ mkieburg@physik.uni-bielefeld.de

[1] C. Chatfield, The Analysis of Time Series: An Introduction, sixth ed. (Chapman and Hall/CRC, 2003) iSBN: 1-58488-317-0.

[2] E. R. Kanasewich, Time Sequence Analysis in Geophysics, 3rd ed. (The University of Alberta Press, Edmonton, Alberta, Canada, 1974) iSBN : 0888640749.

[3] A. M. Tulino and S. Verdu, Random Matrix Theory and Wireless Communications, Foundations and Trends Com. and Inf. Th. (now Publisher Inc, 2004) iSBN:9781933019000.

[4] R. Gnanadesikan, Methods for Statistical Data Analysis of Multivariate Oberservations, second edition ed. (John Wiley \& Sons, 1997).

[5] V. Barnett and T. Lewis, Outliers in Statistical Data, first edition ed. (John Wiley \& Sons, 1980).

[6] Vinayak and A. Pandey, Phys. Rev. E 81, 036202 (2010).

[7] S. Abe and N. Suzuki, "Universal and nonuniversal distant regional correlations in seismicity: Randommatrix approach," ePrint (2009), arXiv:physics.geoph/0909.3830.

[8] M. Müller, G. Baier, A. Galka, U. Stephani, and H. Muhle, Phys. Rev. E 71, 046116 (2005).

[9] P. Šeba, Phys. Rev. Lett. 91, 198104 (2003).

[10] M. S. Santhanam and P. K. Patra, Phys. Rev. E 64, 016102 (2001).

[11] L. Laloux, P. Cizeau, J.-P. Bouchaud, and M. Potters, Phys. Rev. Lett. 83, 1467 (1999).

[12] V. Plerou, P. Gopikrishnan, B. Rosenow, L. A. N. Amaral, T. Guhr, and H. E. Stanley, Phys. Rev. E 65, 066126 (2002).

[13] M. R. Gardner and W. R. Ashby, Nature 228, 784 (1970)

[14] R. M. May, Nature 238, 413414 (1972).

[15] S. N. Majumdar and G. Schehr, Journal of Statistical Mechanics: Theory and Experiment 2014, P01012 (2014).

[16] E. Pianka, Evolutionary Ecology (Eric R. Pianka, 2011).

[17] M. Feinberg, "Lectures on chemical reaction networks,"
(1979), (lecture notes).

[18] A. Stefano and T. Si, Nature 483, 205 (2012).

[19] T. W. Anderson, An Introduction to Multivariate Statistical Analysis, edited by 3rd (Wiley, 2003).

[20] R. J. Muirhead, Aspects of Multivariate Statistical Theory (Published at Wiley Intersience, 2005).

[21] I. M. Johnstone, eprint : arXiv:math/0611589 (2006).

[22] I. M. Johnstone, The Annals of Statistics 29, 295 (2001)

[23] A. Edelman, Math. Comp 58, 185 (1992).

[24] A. Edelman, SIAM Journal on Matrix Analysis and Applications 9, 543 (1988).

[25] Y. Zeng and Y.-C. Liang, Trans. Comm. 57, 1784 (2009).

[26] Y. Zeng and Y.-C. Liang, in Personal, Indoor and Mobile Radio Communications, 200\%. PIMRC 200\%. IEEE 18th International Symposium on (2007) pp. 1-5.

[27] L. Cardoso, M. Debbah, P. Bianchi, and J. Najim, in Wireless Pervasive Computing, 2008. ISWPC 2008. 3rd International Symposium on (2008) pp. 334-338.

[28] L. Wei and O. Tirkkonen, in Personal, Indoor and Mobile Radio Communications, 2009 IEEE 20th International Symposium on (2009) pp. 2295-2299.

[29] F. Penna, R. Garello, D. Figlioli, and M. Spirito, in Cognitive Radio Oriented Wireless Networks and Communications, 2009. CROWNCOM '09. 4th International Conference on (2009) pp. 1-5.

[30] F. Penna and R. Garello, CoRR abs/0907.1523 (2009).

[31] G. Burel, in In Proc. of the WSEAS Int. Conf. on Signal, Speech and Image Processing (ICOSSIP (2002).

[32] C.-N. Chuah, D. Tse, J. Kahn, and R. Valenzuela, Information Theory, IEEE Transactions on 48, 637 (2002).

[33] E. Visotsky and U. Madhow, in Space-time precoding with imperfect feedback (IEEE eXpress Conference Publishing, Sorrento, Italy, 2000) pp. 312-.

[34] L. Wasserman, All of Statistics: A Concise Course in Statistical Inference (Springer, 2003).

[35] H. Markowitz, Portfolio Selection: Efficient Diversification of Investments (J. Wiley and Sons, 1959).

[36] Vinayak, T. Prosen, B. Buca, and T. H. Seligman, "Correlation matrices at the phase transition of the Ising model," (2014), arXiv:1403.7218 [math-ph]

[37] C. Itzykson and J. . B. Zuber, J. Math. Phys. 21, 411 (1980).

[38] A. Balantekin, Phys. Rev. D 62, 085017 (2000).

[39] S. Simon, A. Moustakas, and L. Marinelli, Information Theory, IEEE Transactions on 52, 5336 (2006)

[40] T. Wirtz, M. Kieburg, and T. Guhr, (to be published).

[41] T. Wirtz and T. Guhr, Phys. Rev. Lett. 111, 094101 (2013).

[42] T. Wirtz and T. Guhr, Journal of Physics A: Mathematical and Theoretical 47, 075004 (2014).

[43] Y. V. Fyodorov, Nucl.Phys. B 621, 643 (2002).

[44] M. Kieburg, J. Grönqvist, and T. Guhr, J. Phys. A 42, 275205 (2009).

[45] A. Edelman, Linear Algebra and its Applications 159, 55 (1991).

[46] T. Wilke, T. Guhr, and T. Wettig, Phys.Rev. D 57, 6486 (1998).

[47] P. Forrester, Nuclear Physics B 402, 709 (1993).

[48] P. H. Damgaard and S. M. Nishigaki, Phys. Rev. D 63, 045012 (2001).

[49] K. Johansson, Communications in Mathematical Physics 209, 437 (2000)

[50] A. Soshnikov, Journal of Statistical Physics 108, 1033 (2002) 
[51] N. E. Karoui, "On the largest eigenvalue of wishart matrices with identity covariance when $\mathrm{n}, \mathrm{p}$ and $\mathrm{p} / \mathrm{n}$ tend to infinity," arXiv:math/0309355 (2003).

[52] N. E. Karoui, Ann. Probab. 34, 2077 (2006).

[53] P. Vivo, S. N. Majumdar, and O. Bohigas, Journal of Physics A: Mathematical and Theoretical 40, 4317 (2007)

[54] P. Deift, A. Its, and I. Krasovsky, Communications in Mathematical Physics 278, 643 (2008)

[55] D. Wang, The Annals of Probability 37, pp. 1273 (2009).

[56] O. Feldheim and S. Sodin, Geometric and Functional Analysis 20, 88 (2010)

[57] E. Katzav and I. Pérez Castillo, Phys. Rev. E 82, 040104 (2010)

[58] G. Akemann and P. Vivo, Journal of Statistical Mechanics: Theory and Experiment 2011, P05020 (2011)
[59] C. A. Tracy and H. Widom, Communications in Mathematical Physics 159, 151 (1994).

[60] C. A. Tracy and H. Widom, Physics Letters B 305, 115 (1993).

[61] C. A. Tracy and H. Widom, Communications in Mathematical Physics 177, 727 (1996).

[62] J. Baik, G. B. Arous, and S. Péché, Ann. Probab. Ann. Probab., 1643 (2005).

[63] N. El Karoui, The Annals of Probability 35, 663 (2007).

[64] M. Y. Mo, Communications on Pure and Applied Mathematics 65, 1528 (2012).

[65] A. Bloemendal and B. Virág, Probability Theory and Related Fields 156, 795 (2013)

[66] A. Knowles and J. Yin, "Anisotropic local laws for random matrices," Arxiv: math.PR/1410.3516 (2014). 